\section{Ultrafeiner Staub verstärkt Allergien}

\author{
Feinstaub führt zu einer Belastung der Atemwege und des \\ kardiovaskulären Systems. Der Stellenwert ultrafeiner Koh- \\ lenstoffpartikel mit einem Durchmesser von unter 0,1 $\mu \mathrm{m}$ \\ wird dabei möglicherweise noch unterschätzt.
}

\begin{abstract}
A ufgrund ihres sehr geringen Durchmessers gelangen ultrafeine Partikel (UFP) nach Inhalation schnell in die Lungenperipherie und das pulmonale Interstitium. Verschiedene Studien weisen darauf hin, dass Patienten mit allergischem Asthma empfindlicher reagieren, wenn die Umgebungsluft mit UFP angereichert ist. Experimentelle Untersuchungen, die diese Hypothese belegen, gab es bislang aber nicht.

Vor diesem Hintergrund setzte ein Team des Zentrums Allergie und Umwelt der Technischen Universität München Mäuse einer UFP-Belastung von $526 \mu \mathrm{g} / \mathrm{m}^{3}$ aus. Dabei wurden sowohl gegen Ovalbumin sensibilisierte Mäuse verwendet als auch nicht allergische Tiere als Kontrolle. Die Partikelexposition erfolgte vor bzw. nach einer Allergenprovokation mit Ovalbumin. Die sensibilisierten Mäuse zeigten im Vergleich zur Kontrollgruppe nach Provokation eine
\end{abstract}

signifikante und deutliche $\mathrm{Zu}$ nahme von Eosinophilen und Neutrophilen sowie von Interleukin-4, -5 und -13 in der Bronchiallavage. Dies war besonders dann der Fall, wenn die Mäuse vor der Allergenexposition UFP ausgesetzt waren. Mäuse, die erst nach Allergenexposition mit den Partikeln in Kontakt kamen, zeigten in der Lavage nur eine moderate und verzögerte allergische Reaktion. Die verstärkte allergische Reaktion bei zuvor UFP-exponierten Mäusen war auch histologisch als erhöhte Mukusproduktion sowie als peribronchiale und -vaskuläre Entzündung und klinisch als bronchiale Hyperreagibilität in der Ganzkörperplethysmografie nachweisbar.

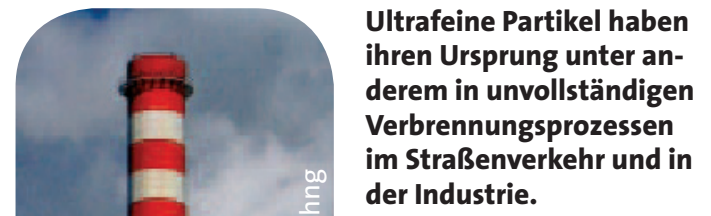

Fazit: In dieser experimentellen Studie führte die Exposition von Ovalbumin-sensibilisierten Mäusen mit ultrafeinen Karbonpartikeln zu einer deutlich verstärkten Reaktion nach Allergenprovokation. Die Vermutung liegt nahe, dass auch allergische Patienten, die einer hohen Partikelbelastung ausgesetzt sind, heftiger auf die entsprechenden Allergene reagieren. af

Alessandrini $\mathrm{F}$ et al. Effects of ultrafine carbon particle inhalation on allergic inflammation of the lung. J Allergy Clin Immunol 2006; 117: 824-30

\section{Anisakis-Allergie auffallend häufig}

\author{
Nahrungsmittelallergien gegen Fisch sind bekannt. Doch wie ver- \\ breitet sind Allergien gegen Fischparasiten? Südafrikanische Forscher \\ untersuchten im klinischen und tierexperimentellen Ansatz die \\ Sensibilisierung gegen den Fischparasiten Anisakis.
}

든 in Allergologenteam aus Kapstadt untersuchte 578 Arbeiter aus Fischfabriken. Der Anteil der Beschäftigten, die im Pricktest auf Anisakis reagierten, war mit $8 \%$ erstaunlich hoch. Auf Fischeiweiß, ein klassisches Nahrungsmittelallergen, war dagegen der Pricktest nur bei $6 \%$ positiv. Das Risiko einer Sensibilisierung gegen Anisakis jedoch nicht gegen Fisch - war bei hohem Fischkonsum signifikant höher. Die Arbeiter, bei denen die Sensibilisierung zu einer klinisch manifesten All- ergie geführt hatte, klagten über Symptome nach Konsum, aber auch nach Hautkontakt mit Anisakis-infiziertem Fisch.

Anschließend sollte die Sensibilisierung im Mausmodell nachvollzogen werden: Dazu wurden Mäuse zunächst mit Anisakis-pergreffii-Larven intraperitoneal infiziert. Splenozyten, die von diesen Mäusen kultiviert worden waren, zeigten nach Stimulation eine signifikant höhere Produktion von Interleukin-4, -5, -9 und -13 als Splenozyten- kulturen von Kontrolltieren. Mäuse, die mit den Larven infiziert worden waren, zeigten bei wiederholter Infektion einen deutlichen Anstieg von spezifischem $\operatorname{IgE}$ und $\mathrm{IgG}_{1}$. Erhielten die Mäuse im weiteren Verlauf AnisakisProtein oral verabreicht, reagierten sie mit Inaktivität, heftigem Kratzen und Durchfall.

Fazit: Sensibilisierungen und Allergien gegen den Fischparasiten Anisakis sind bei Arbeitern in der Fischindustrie häufiger als gegen Fischeiweiß. Die Sensibilisierungsrate hängt dabei vom Fischkonsum ab.

Nieuwenhuizen $\mathbf{N}$ et al. Exposure to the fish parasite Anisakis causes allergic airway hyperreactivity and dermatitis. J Allergy Clin Immunol 2006; 117: 1098-105. 